\title{
ON THE NAVIER-STOKES EQUATIONS WITH TEMPERATURE-DEPENDENT TRANSPORT COEFFICIENTS
}

\author{
EDUARD FEIREISL AND JOSEF MÁLEK
}

Received 13 September 2005; Revised 2 April 2006; Accepted 3 April 2006

We establish long-time and large-data existence of a weak solution to the problem describing three-dimensional unsteady flows of an incompressible fluid, where the viscosity and heat-conductivity coefficients vary with the temperature. The approach reposes on considering the equation for the total energy rather than the equation for the temperature. We consider the spatially periodic problem.

Copyright (C) 2006 E. Feireisl and J. Málek. This is an open access article distributed under the Creative Commons Attribution License, which permits unrestricted use, distribution, and reproduction in any medium, provided the original work is properly cited.

\section{Introduction}

Viscosity plays an important role in most of the problems of fluid dynamics. In the simplest case when the viscous stress tensor $\mathbb{S}$ is a linear function of the fluid velocity gradient, the principle of material frame indifference yields

$$
\mathbb{S}=\mu\left(\nabla_{x} \mathbf{u}+\nabla_{x} \mathbf{u}^{t}-\frac{2}{3} \operatorname{div} \mathbf{u I}\right)+\zeta \operatorname{div} \mathbf{u} \mathbb{I}
$$

where $\mathbf{u}$ is the fluid velocity, and $\mu$ and $\zeta$ are scalar quantities termed the shear viscosity coefficient and the bulk viscosity coefficient, respectively.

If the fluid is considered incompressible, the velocity is subject to the well-known constraint

$$
\operatorname{div} \mathbf{u}=0
$$

If, in addition, the fluid is homogeneous, the mass balance equation results in the fact that the fluid density is everywhere equal to a positive constant denoted by $\bar{\varrho}$. The motion of the fluid is then described by the equation representing the balance of linear momentum that takes the form

$$
\bar{\varrho}\left(\partial_{t} \mathbf{u}+\mathbf{u} \cdot \nabla_{x} \mathbf{u}\right)+\nabla_{x} p=\operatorname{div} \mathbb{S}+\bar{\varrho} \mathbf{f},
$$

where $p$ is the pressure, and $\mathbf{f}$ denotes a given external force density. 
2 NSEs with temperature-dependent transport coefficients

If, moreover, the fluid is heat conductive, the above equations have to be supplemented with the equation

$$
c_{V} \bar{\varrho}\left(\partial_{t} \theta+\mathbf{u} \cdot \nabla_{x} \theta\right)+\operatorname{div} \mathbf{q}=\mathbb{S}: \nabla_{x} \mathbf{u},
$$

where $\theta$ stands for the absolute temperature, $c_{V}$ is the specific heat, and $\mathbf{q}$ is the heat flux obeying, conformably to (1.1), Fourier's law,

$$
\mathbf{q}=-\kappa \nabla_{x} \theta
$$

with the heat conductivity coefficient $\kappa$. The so-called dissipation function $\mathbb{S}: \nabla_{x} \mathbf{u}$, omitted frequently in many mathematical models, represents the irreversible transfer of the mechanical energy into heat.

The simplest situation to consider, as a paradigm of the above-described problem, is that of a layer of depth $h>0$ placed between two horizontal boundaries located at $x_{3}=0$ and $x_{3}=h$, where we prescribe the no-stick boundary conditions for the velocity:

$$
\begin{gathered}
u_{3}\left(t, x_{1}, x_{2}, 0\right)=u_{3}\left(t, x_{1}, x_{2}, h\right)=0 \quad \forall\left(x_{1}, x_{2}\right) \in \mathbb{R}^{2}, \\
\left\{\begin{array}{c}
\left(\mathbb{S}\left(t, x_{1}, x_{2}, 0\right)[0,0,-1]\right) \times[0,0,-1]=0 \\
\left(\mathbb{S}\left(t, x_{1}, x_{2}, h\right)[0,0,1]\right) \times[0,0,1]=0
\end{array}\right\} \quad \forall\left(x_{1}, x_{2}\right) \in \mathbb{R}^{2},
\end{gathered}
$$

and the no-flux boundary conditions for $\theta$ :

$$
\partial_{x_{3}} \theta\left(t, x_{1}, x_{2}, 0\right)=\partial_{x_{3}} \theta\left(t, x_{1}, x_{2}, h\right)=0 \quad \forall\left(x_{1}, x_{2}\right) \in \mathbb{R}^{2} .
$$

Moreover, all quantities we deal with are supposed to be spatially periodic in $x_{1}$ and $x_{2}$ with periods $h_{1}$ and $h_{2}$, respectively.

The main objective of the present paper is to study the physically relevant case, where the transport coefficients $\mu$ and $\kappa$ are effective functions of the absolute temperature $\theta$. More specifically, we suppose that

$$
\mu, \kappa \in C^{2}[0, \infty), \quad 0<\underline{\mu} \leq \mu(\theta) \leq \bar{\mu}, 0<\underline{\kappa} \leq \kappa(\theta) \leq \bar{\kappa} \quad \forall \theta \in[0, \infty) .
$$

Clearly, the bulk viscosity becomes irrelevant under the incompressibility condition (1.2).

It is easy to observe (cf. Ebin [5]) that the boundary conditions (1.6)-(1.7) can be conveniently reformulated in terms of spatial periodicity on $\mathbb{R}^{3}$ supplemented with additional symmetry properties. Taking, for simplicity, $h=\pi, h_{1}=h_{2}=2 \pi$, we can consider the state variables $\mathbf{u}(t, \cdot), \theta(t, \cdot)$ defined on the three-dimensional torus $\mathscr{T}^{3}=[-\pi, \pi]^{3}:=$ $\left\{\left(x_{1}, x_{2}, x_{3}\right) ; x_{i} \in[-\pi, \pi], \forall i=1,2,3\right\}$, where we identify the points $\left(-\pi, x_{2}, x_{3}\right)$ with $\left(\pi, x_{2}, x_{3}\right)$ and so forth, that are $2 \pi$-periodic in $x_{1}$ and $x_{2}$ and satisfy

$$
\begin{gathered}
\left\{\begin{array}{c}
u_{i}\left(t, x_{1}, x_{2}, x_{3}\right)=u_{i}\left(t, x_{1}, x_{2},-x_{3}\right), i=1,2, \\
u_{3}\left(t, x_{1}, x_{2}, x_{3}\right)=-u_{3}\left(t, x_{1}, x_{2},-x_{3}\right)
\end{array}\right\} \quad \forall\left(x_{1}, x_{2}, x_{3}\right) \in \mathscr{T}^{3}, \\
\theta\left(t, x_{1}, x_{2}, x_{3}\right)=\theta\left(t, x_{1}, x_{2},-x_{3}\right) \quad \forall\left(x_{1}, x_{2}, x_{3}\right) \in \mathscr{T}^{3} .
\end{gathered}
$$


Moreover, without loss of generality, we set $C_{V} \bar{\varrho}=1$ and, for the sake of simplicity, $\mathbf{f} \equiv \mathbf{0}$.

The existence of global-in-time solutions for system (1.2)-(1.4) supplemented with the initial data

$$
\mathbf{u}(0, x)=\mathbf{u}_{0}(x), \quad \theta(0, x)=\theta_{0}(x), \quad x \in \mathscr{T}^{3}
$$

is an open problem, even in the class of the weak solutions introduced for the standard Navier-Stokes system by Leray [9]. The main stumbling block is represented by the dissipative term

$$
\mathbb{S}: \nabla_{x} \mathbf{u}=\frac{\mu(\theta)}{2}\left|\nabla_{x} \mathbf{u}+\nabla_{x} \mathbf{u}^{t}\right|^{2}
$$

being the only source of a priori estimates on the velocity gradient $\nabla_{x} \mathbf{u}$, and, at the same time, a quantity which is only weakly lower semicontinuous in $\nabla_{x} \mathbf{u}$. Consequently, (1.4) has to be replaced by the inequality

$$
\partial_{t} \theta+\mathbf{u} \cdot \nabla_{x} \theta+\operatorname{div} \mathbf{q} \geq \mathbb{S}: \nabla_{x} \mathbf{u}
$$

related clearly to the local (kinetic) energy inequality

$$
\partial_{t}\left(\frac{1}{2}|\mathbf{u}|^{2}\right)+\operatorname{div}\left(\left(\frac{1}{2}|\mathbf{u}|^{2}+p\right) \mathbf{u}\right)-\operatorname{div}(\mathbb{S u})+\mathbb{S}: \nabla_{x} \mathbf{u} \leq 0
$$

that is known to hold for the so-called suitable weak solutions of the Navier-Stokes system introduced by Caffarelli et al. [1]. Of course, such a problem does not occur when there are better a priori estimates on the velocity gradient as it is the case for, say, some non-Newtonian fluids (see Consiglieri [2] or Nečas and Roubíček [12]) or in two space dimensions (see Consiglieri et al. [3]). Note also that the issue of smoothness of the solutions to an approximative problem (neglecting the convective terms in (1.3) and (1.4)) is addressed by Shilkin in [13].

The question whether or not (1.14) may hold as a strict inequality is completely open and has been discussed even for the classical Navier-Stokes and Euler equations by many authors (see Duchon and Robert [4], Eyink [6], and Nagasawa [11] for the most recent results). The problem is nontrivial, and the correct answer would certainly represent a highly desired piece of information in the mathematical theory of the Navier-Stokes system. Here, it seems worth-noting that a strict inequality in (1.14) would definitely imply the same for (1.13) as the total energy of the system has to be conserved.

Our strategy is to replace (1.4) by the balance of total energy that has clear physical background. Similarly, as the notion of local energy inequality introduced in [1], the total energy balance requires to know the pressure that is frequently omitted in the analysis of incompressible fluids (by restricting to the spaces of test functions that are free of 
4 NSEs with temperature-dependent transport coefficients

divergence). The resulting system consists of three equations:

$$
\begin{gathered}
\partial_{t} \mathbf{u}+\operatorname{div}(\mathbf{u} \otimes \mathbf{u})+\nabla_{x} p=\operatorname{div}\left(\mu(\theta)\left(\nabla_{x} \mathbf{u}+\nabla_{x} \mathbf{u}^{t}\right)\right), \\
\partial_{t}\left(\frac{1}{2}|\mathbf{u}|^{2}+\theta\right)+\operatorname{div}\left(\left(\frac{1}{2}|\mathbf{u}|^{2}+p+\theta\right) \mathbf{u}\right)-\Delta \mathscr{K}(\theta)=\operatorname{div}\left(\mu(\theta)\left(\nabla_{x} \mathbf{u}+\nabla_{x} \mathbf{u}^{t}\right) \mathbf{u}\right), \\
\operatorname{div} \mathbf{u}=0,
\end{gathered}
$$

that can be supplemented with an "entropy" inequality:

$$
\partial_{t} \theta+\operatorname{div}(\theta \mathbf{u})-\Delta \mathscr{K}(\theta) \geq \frac{\mu(\theta)}{2}\left|\nabla_{x} \mathbf{u}+\nabla_{x} \mathbf{u}^{t}\right|^{2},
$$

where we have set

$$
\mathscr{K}(\theta)=\int_{0}^{\theta} \kappa(z) \mathrm{d} z
$$

In the framework of weak (distributional) solutions, inequality (1.18) may be viewed as an extra admissibility condition.

The reason for writing $\kappa(\theta) \nabla_{x} \theta=\nabla_{x} \mathscr{K}(\theta)$ is due to the lack of suitable a priori estimates to render the quantity $\kappa \nabla_{x} \theta$ locally integrable (cf. (1.24) below). On the other hand, the weak formulation allows us to write

$$
\int_{\mathscr{T}^{3}} \Delta \mathscr{K}(\theta) \varphi \mathrm{d} x=\int_{\mathscr{T}^{3}} \mathscr{K}(\theta) \Delta \varphi \mathrm{d} x
$$

where the right-hand side makes sense for any "test" function $\varphi \in C^{2}\left(\mathscr{T}^{3}\right)$.

The main objective of the present paper is to establish the following existence result.

THeorem 1.1. Let $\mu$ and $\kappa$ satisfy hypothesis (1.8). Furthermore suppose that

$$
\mathbf{u}_{0} \in L^{2}\left(\mathscr{T}^{3} ; \mathbb{R}^{3}\right), \quad \theta_{0} \in L^{1}\left(\mathscr{T}^{3}\right)
$$

enjoy the symmetry properties specified in (1.9), (1.10), and that

$$
\operatorname{div} \mathbf{u}_{0}=0, \quad \text { ess } \inf _{x \in \mathscr{T}^{3}} \theta_{0}(x)>0 .
$$

Then there exist functions $(\mathbf{u}, \theta, p)$ such that

$$
\begin{gathered}
\mathbf{u} \in L^{2}\left(0, T ; W^{1,2}\left(\mathscr{T}^{3} ; \mathbb{R}^{3}\right)\right) \cap C\left([0, T] ; L_{\text {weak }}^{2}\left(\mathscr{T}^{3} ; \mathbb{R}^{3}\right)\right), \\
\left\{\begin{array}{c}
\theta \in L^{\infty}\left(0, T ; L^{1}\left(\mathscr{T}^{3}\right)\right), \theta(t, x)>0 \text { for a.a. }(t, x) \in(0, T) \times \mathscr{T}^{3}, \\
\left.\theta^{\alpha / 2} \in L^{2}\left(0, T ; W^{1,2}\left(\mathscr{T}^{3}\right)\right) \text { for any (positive }\right) \alpha<1,
\end{array}\right\} \\
t \longmapsto \int_{\mathscr{T}^{3}}\left(\frac{1}{2}|\mathbf{u}(t)|^{2}+\theta(t)\right) \varphi d x \in C([0, T]) \quad \forall \varphi \in C(\Omega) \\
p \in L^{5 / 3}\left((0, T) \times \mathscr{T}^{3}\right),
\end{gathered}
$$


belonging to the symmetry class (1.9), (1.10), and satisfying system (1.15)-(1.18) in $\mathscr{D}^{\prime}$ $\left((0, T) \times \mathscr{T}^{3}\right)$, together with the initial conditions

$$
\begin{gathered}
\mathbf{u}(0)=\mathbf{u}_{0}, \quad \frac{1}{2}|\mathbf{u}(0)|^{2}+\theta(0)=\frac{1}{2}\left|\mathbf{u}_{0}\right|^{2}+\theta_{0}, \\
\operatorname{ess} \liminf _{t \rightarrow 0+} \int_{\mathscr{T}^{3}} \theta(t) \varphi \mathrm{d} x \geq \int_{\mathscr{T}^{3}} \theta_{0} \varphi \mathrm{d} x \quad \text { for any } \varphi \in \mathscr{D}\left(\mathscr{T}^{3}\right), \varphi \geq 0 .
\end{gathered}
$$

The paper is organized as follows. In Section 2, we introduce a family of approximate problems solvable by the standard techniques. Following Leray's original idea, we use smoothing operators applied to the velocity field appearing in the convective term. At the same time, the (modified) momentum equation is solved via the standard FaedoGalerkin approximations while the temperature is expressed through (1.4).

In Section 3, we derive uniform estimates on the sequence of approximate solutions introduced in Section 2. In particular, these estimates prevent the approximate solutions to "blow up" in a finite time. From this point of view, the most delicate quantity to deal with seems to be the temperature $\theta$, for which the energy equation (1.4) has to be reformulated in the spirit of the theory of renormalized solutions, where the desired estimates are obtained through interpolation techniques.

As a next step, we obtain the "second" level approximate solutions resulting from the Faedo-Galerkin scheme (see Section 4). These quantities are shown to solve a system similar to (1.15)-(1.18), where the velocity field appearing in the convective terms in (1.15), (1.16) is still replaced by its regularization.

Finally, getting rid of the regularized terms we complete the proof of Theorem 1.1 in Section 5 .

\section{The Faedo-Galerkin approximation scheme}

Given the periodic boundary conditions, it is convenient to use the Faedo-Galerkin approximation scheme in order to solve (1.15), where the temperature is obtained through the heat equation (1.4). To this end, we introduce the Fourier series representation of a function $v$ :

$$
v(t, x)=\sum_{\mathbf{k} \in \mathbb{Z}^{3}}[v]_{\mathbf{k}}(t) \exp (\mathbf{i k} \cdot x) \quad \text { where }[v]_{\mathbf{k}}(t)=\frac{1}{\left|\mathscr{T}^{3}\right|} \int_{\mathscr{T}^{3}} v(t, y) \exp (-\mathbf{i k} \cdot y) d y .
$$

Accordingly, Helmholtz's projection $\mathscr{H}$ onto the space of solenoidal (divergenceless) functions can be written as

$$
[\mathscr{H}[\mathbf{v}]]_{\mathbf{k}}=[\mathbf{v}]_{\mathbf{k}}-\frac{\mathbf{k}}{|\mathbf{k}|^{2}} \mathbf{k} \cdot[\mathbf{v}]_{\mathbf{k}}, \quad \mathbf{k} \in \mathbb{Z}^{3}, \mathbf{v} \in L^{2}\left(\mathscr{T}^{3} ; \mathbb{R}^{3}\right) .
$$

Set

$$
X_{n, \operatorname{div}}=\left\{\mathbf{w}\left|\mathscr{H}[\mathbf{w}]=\mathbf{w},[\mathbf{w}]_{\mathbf{k}}=0 \forall \mathbf{k},\right| \mathbf{k} \mid>n\right\}
$$


$6 \quad$ NSEs with temperature-dependent transport coefficients

For a given $M>0$, we introduce the regularizing operators

$$
[v]_{|\mathbf{k}| \leq M}=\sum_{|\mathbf{k}| \leq M}[v]_{\mathbf{k}} \exp (i \mathbf{k} \cdot x)
$$

and look for the approximate fields $\mathbf{u}_{n} \in C\left(\left[0, T_{n}\right] ; X_{n \text {,div }}\right)$ and $\theta_{n}$ that are determined through the system of equations

$$
\begin{aligned}
& \frac{d}{d t} \int_{\mathscr{T}^{3}} \mathbf{u}_{n} \cdot \mathbf{w} \mathrm{d} x=-\int_{\mathscr{T}^{3}} \mu\left(\theta_{n}\right)\left(\nabla_{x} \mathbf{u}_{n}+\nabla_{x} \mathbf{u}_{n}^{t}\right): \nabla_{x} \mathbf{w} \mathrm{d} x \\
&+\int_{\mathscr{T}^{3}}\left(\mathbf{u}_{n} \otimes\left[\mathbf{u}_{n}\right]_{|\mathbf{k}| \leq M}\right): \nabla_{x} \mathbf{w} \mathrm{d} x \text { to be valid } \quad \forall \mathbf{w} \in X_{n, \text { div }}, \\
& \partial_{t} \theta_{n}+\operatorname{div}\left(\theta_{n} \mathbf{u}_{n}\right)-\operatorname{div}\left(\kappa\left(\theta_{n}\right) \nabla_{x} \theta_{n}\right)=\frac{\mu\left(\theta_{n}\right)}{2}\left|\nabla_{x} \mathbf{u}_{n}+\nabla_{x} \mathbf{u}_{n}^{t}\right|^{2},
\end{aligned}
$$

completed by the set of initial conditions

$$
\begin{gathered}
\mathbf{u}_{n}(0)=\mathbf{u}_{n, 0}=\sum_{|\mathbf{k}| \leq n}\left[\mathbf{u}_{0}\right]_{\mathbf{k}} \exp (i \mathbf{k} \cdot x) \longrightarrow \mathbf{u}_{0} \quad \text { in } L^{2}\left(\mathscr{T}^{3}\right), \\
\theta_{n}(0)=\theta_{n, 0},
\end{gathered}
$$

with $\theta_{n, 0} \in C^{\infty}\left(\mathscr{T}^{3}\right)$ satisfying $(1.10)$,

$$
\inf _{n \in N, x \in \mathscr{T}^{3}} \theta_{n, 0}>0, \quad \theta_{n, 0} \longrightarrow \theta_{0} \quad \text { in } L^{1}\left(\mathscr{T}^{3}\right) .
$$

As for solvability of the parabolic equation (2.6), we report (and sketch the proof of) the following (classical) result.

Lemma 2.1. Given $\mathbf{u}_{n} \in C\left([0, T] ; X_{n, \text { div }}\right), \theta_{n, 0} \in L^{\infty}\left(\mathscr{T}^{3}\right)$ fulfilling (2.9), there exists a unique function

$$
\theta_{n} \in L^{\infty}\left((0, T) \times \mathscr{T}^{3}\right) \cap L^{2}\left(0, T ; W^{1,2}\left(\mathscr{T}^{3}\right)\right)
$$

solving (2.6) in $\mathscr{D}^{\prime}\left((0, T) \times \mathscr{T}^{3}\right)$ and satisfying the initial condition (2.8).

Furthermore,

$$
\theta_{n}(t, x) \geq \mathrm{ess} \inf _{y \in \mathscr{T}^{3}} \theta_{n, 0}(y) \quad \text { for a.a. } t \in(0, T), x \in \mathscr{T}^{3},
$$

and a "renormalized" equation

$$
\begin{aligned}
& \partial_{t} H\left(\theta_{n}\right)+\operatorname{div}\left(H\left(\theta_{n}\right) \mathbf{u}_{n}\right)-\operatorname{div}\left(H^{\prime}\left(\theta_{n}\right) \kappa\left(\theta_{n}\right) \nabla_{x} \theta_{n}\right) \\
& \quad=\frac{1}{2} H^{\prime}\left(\theta_{n}\right) \mu\left(\theta_{n}\right)\left|\nabla_{x} \mathbf{u}_{n}+\nabla_{x} \mathbf{u}_{n}^{t}\right|^{2}-H^{\prime \prime}\left(\theta_{n}\right) \kappa\left(\theta_{n}\right)\left|\nabla_{x} \theta_{n}\right|^{2}
\end{aligned}
$$

holds in $\mathscr{D}^{\prime}\left((0, T) \times \mathscr{T}^{3}\right)$ for any $H \in C^{2}[0, \infty)$. 
Proof. (i) To begin with, observe that any distributional solution of (2.6) belongs to the class $C\left([0, T] ; L^{2}\left(\mathscr{T}^{3}\right)\right)$; whence (2.8) makes sense.

(ii) Now it is easy to see that two arbitrary distributional solutions $\theta_{n}^{1}, \theta_{n}^{2}$ of (2.6) satisfy

$$
\begin{aligned}
& \frac{1}{2}\left\|\theta_{n}^{1}\left(t_{2}\right)-\theta_{n}^{2}\left(t_{2}\right)\right\|_{L^{2}\left(\mathscr{T}^{3}\right)}^{2}+c_{1} \int_{t_{1}}^{t_{2}} \int_{\mathscr{T}^{3}}\left|\nabla_{x} \theta_{n}^{1}-\nabla_{x} \theta_{n}^{2}\right|^{2} \mathrm{~d} x \mathrm{~d} t \\
& \quad \leq \frac{1}{2}\left\|\theta_{n}^{1}\left(t_{1}\right)-\theta_{n}^{2}\left(t_{1}\right)\right\|_{L^{2}\left(\mathscr{T}^{3}\right)}^{2}+c_{2} \int_{t_{1}}^{t_{2}} \int_{\mathscr{T}^{3}}\left|\theta_{n}^{1}-\theta_{n}^{2}\right|^{2} \mathrm{~d} x \mathrm{~d} t \quad \text { for any } 0 \leq t_{1}<t_{2} \leq T,
\end{aligned}
$$

where $c_{1}, c_{2}$ depend only on $\mathbf{u}_{n}$, ess sup $\left|\theta_{n}^{i}\right|, i=1,2$, ess sup $\left|\nabla \theta_{n}^{2}\right|$, and the structural constants $\mu, \bar{\mu}, \underline{\kappa}, \bar{\kappa}$ appearing in hypothesis (1.8). In particular, any distributional solution of (2.6) belonging to the class (2.10) is uniquely determined by the initial data.

(iii) In order to establish the existence of solution, we can for example start with approximating the functions $\mu, \kappa$ by smooth ones and regularizing $\mathbf{u}_{n}$ in time to solve the resulting problem with the help of the classical theory (see Ladyzhenskaya et al. [8]).

At this stage, the lower bound claimed in (2.11) as well as an upper bound, depending on $\mathbf{u}_{n}$, ess sup $\left|\theta_{n, 0}\right|$, and $\bar{\mu}$, follow directly from the maximum principle. Furthermore regular solutions obey automatically the (regularized) equation (2.12).

Finally it is easy to show that the solutions of the regularized problems are bounded in the space specified in (2.10) independently of the degree of regularization. Consequently, the Lions-Aubin lemma can be applied in order to show that this sequence admits a limit $\theta_{n}$-the unique solution of (2.6), (2.8) satisfying (2.11), (2.12). The reader may wish to consult Feireisl [7, Section 7.3.2.], or Consiglieri et al. [3] for more details.

By virtue of Lemma 2.1, the absolute temperature $\theta_{n}$ appearing in the second integral on the right-hand side of (2.5) can be expressed through (2.6), (2.8) for any given $\mathbf{u}_{n} \in C\left([0, T] ; X_{n, \text { div }}\right)$. Accordingly, problem (2.5)-(2.8) can be solved via the standard Caratheodory theory, at least on a (possibly) short time interval $\left(0, T_{n}\right)$. Since $\mathbf{u}_{n}$ is continuously differentiable on $\left(0, T_{n}\right)$ we can pick $\mathbf{w}=\mathbf{u}_{n}(t)$ in (2.5) to obtain the identity (that gives rise to the "kinetic" energy balance)

$$
\frac{1}{2} \frac{d}{\mathrm{~d} t} \int_{\mathscr{T}^{3}}\left|\mathbf{u}_{n}\right|^{2} \mathrm{~d} x+\int_{\mathscr{T}^{3}} \frac{\mu\left(\theta_{n}\right)}{2}\left|\nabla_{x} \mathbf{u}_{n}+\nabla_{x} \mathbf{u}_{n}^{t}\right|^{2} \mathrm{~d} x=0
$$

This particularly leads to the estimate

$$
\sup _{t \in\left[0, T_{n}\right)}\left\|\mathbf{u}_{n}(t)\right\|_{L^{2}\left(\mathscr{T}^{3}\right)} \leq\left\|\mathbf{u}_{0}\right\|_{L^{2}\left(\mathscr{T}^{3}\right)} \quad \forall n=1,2, \ldots
$$

that immediately implies that the existence time $T_{n}$ is independent of $n$. More specifically, we can take $T_{n}=T$; whence, keeping $n$ and $M$ fixed, we have established the existence of solutions $\left(\mathbf{u}_{n}, \theta_{n}\right):=\left(\mathbf{u}_{n, M}, \theta_{n, M}\right)$ to the approximative problem (2.5)-(2.8). 
$8 \quad$ NSEs with temperature-dependent transport coefficients

To conclude we make yet another observation. Setting

$$
X_{n}=\left\{\mathbf{w}\left|[\mathbf{w}]_{\mathbf{k}}=0 \forall \mathbf{k},\right| \mathbf{k} \mid>n\right\}
$$

we notice that (2.5) can be written as

$$
\begin{aligned}
\frac{d}{\mathrm{~d} t} \int_{\mathscr{T}^{3}} \mathbf{u}_{n} \cdot \mathbf{w} \mathrm{d} x= & \int_{\mathscr{T}^{3}} p_{n} \operatorname{div} \mathbf{w} \mathrm{d} x+\int_{\mathscr{T}^{3}}\left(\mathbf{u}_{n} \otimes\left[\mathbf{u}_{n}\right]_{|\mathbf{k}| \leq M}\right): \nabla_{x} \mathbf{w} \mathrm{d} x \\
& -\int_{\mathscr{T}^{3}} \mu\left(\theta_{n}\right)\left(\nabla_{x} \mathbf{u}_{n}+\nabla_{x} \mathbf{u}_{n}^{t}\right): \nabla_{x} \mathbf{w} \mathrm{d} x \quad \text { valid } \forall \mathbf{w} \in X_{n},
\end{aligned}
$$

where the approximate pressure term $p_{n}$ is (uniquely) determined through

$$
p_{n}=\left(\nabla_{x} \Delta^{-1} \nabla_{x}\right):\left[\mu\left(\theta_{n}\right)\left(\nabla_{x} \mathbf{u}_{n}+\nabla_{x} \mathbf{u}_{n}^{t}\right)-\left(\mathbf{u}_{n} \otimes\left[\mathbf{u}_{n}\right]_{|\mathbf{k}| \leq M}\right)\right]
$$

Here the inverse Laplace operator $\Delta^{-1}$ is considered on the space of spatially periodic functions with zero mean; whence $\left(\nabla_{x} \Delta^{-1} \nabla_{x}\right)$ may be viewed as a pseudodifferential operator

$$
\left[\left(\nabla_{x} \Delta^{-1} \nabla_{x}\right) v\right]_{\mathbf{k}}=\frac{\mathbf{k} \otimes \mathbf{k}}{|\mathbf{k}|^{2}}[v]_{\mathbf{k}}, \quad \mathbf{k} \in \mathbb{Z}^{3}
$$

In particular, as a direct consequence of the Calderon-Zygmund theory,

$$
\left(\nabla_{x} \Delta^{-1} \nabla_{x}\right): L^{p}\left(\mathscr{T}^{3}\right) \longrightarrow L^{p}\left(\mathscr{T}^{3} ; \mathbb{R}^{3 \times 3}\right) \text { is bounded for any } 1<p<\infty .
$$

\section{Uniform estimates}

Our aim is to derive estimates on the sequence of approximate solutions $\left(\mathbf{u}_{n}, p_{n}, \theta_{n}\right):=$ $\left(\mathbf{u}_{n, M}, p_{n, M}, \theta_{n, M}\right)$ constructed in the preceding section. These estimates are of two types: some of them will be uniform not only with respect to $n$ but also to $M$, while others will be independent of $n$ only. For the estimates of the first group, we will use the absolute positive constant $K$ to bound all of them, while the estimates depending on $M$ will be denoted by $C=C(M)$ independent of $n$.

First of all, by virtue of hypothesis (1.8), relation (2.14) gives rise to the standard energy estimates:

$$
\begin{aligned}
\left\|\mathbf{u}_{n}\right\|_{L^{\infty}\left(0, T ; L^{2}\left(\mathscr{T}^{3} ; \mathbb{R}^{3}\right)\right)} & \leq K, \\
\left\|\mathbf{u}_{n}\right\|_{L^{2}\left(0, T ; W^{1,2}\left(\mathscr{T}^{3} ; \mathbb{R}^{3}\right)\right)} & \leq K .
\end{aligned}
$$

Note that (3.1) and (3.2) imply, using standard interpolation inequalities, that

$$
\left\|\mathbf{u}_{n}\right\|_{L^{10 / 3}\left(0, T ; L^{10 / 3}\left(\mathcal{T}^{3} ; \mathbb{R}^{3}\right)\right)} \leq K
$$


Consequently,

$$
\left\|\mathbf{u}_{n} \otimes\left[\mathbf{u}_{n}\right]_{|\mathbf{k}| \leq M}\right\|_{L^{5 / 3}\left(0, T ; L^{5 / 3}\left(\mathscr{T}^{3} ; \mathbb{R}^{3} \times \mathbb{R}^{3}\right)\right)} \leq K
$$

Since the truncated function $\left[\mathbf{u}_{n}\right]_{|\mathbf{k}| \leq M}$ is for $M$ fixed smooth, we also have

$$
\left\|\mathbf{u}_{n} \otimes\left[\mathbf{u}_{n}\right]_{|\mathbf{k}| \leq M}\right\|_{L^{10 / 3}\left(0, T ; L^{10 / 3}\left(\mathscr{T}^{3} ; \mathbb{R}^{3} \times \mathbb{R}^{3}\right)\right)} \leq C(M) .
$$

Next, we come to the estimates for the pressures $p_{n}:=p_{n, M}$. In virtue of $(2.20)$ and estimates (3.2), (3.4), (3.5), it follows from formula (2.18) that

$$
\begin{aligned}
\left\|p_{n}\right\|_{L^{5 / 3}\left(0, T ; L^{5 / 3}\left(\mathcal{T}^{3} ; \mathbb{R}\right)\right)} & \leq K, \\
\left\|p_{n}\right\|_{L^{2}\left(0, T ; L^{2}\left(\mathcal{T}^{3} ; \mathbb{R}\right)\right)} & \leq C(M) .
\end{aligned}
$$

Combining (3.2), (3.4), (3.6) with (2.17), we deduce that

$$
\left\|\partial_{t} \mathbf{u}_{n}\right\|_{L^{5 / 2}\left(0, T ; W^{\left.-1,5 / 2\left(\mathscr{T}^{3} ; \mathbb{R}^{3}\right)\right)}\right.} \leq K
$$

and, similarly, relations (3.2), (3.5), and (3.7) yield

$$
\left\|\partial_{t} \mathbf{u}_{n}\right\|_{L^{2}\left(0, T ; W^{\left.-1,2\left(\mathscr{T}^{3} ; \mathbb{R}^{3}\right)\right)}\right.} \leq C(M) .
$$

Finally, we derive suitable estimates for the temperatures $\theta_{n}:=\theta_{n, M}$. It follows from (3.2) and hypothesis (1.8) that the source term on the right-hand side of (2.6) is bounded in $L^{1}\left((0, T) \times \mathscr{T}^{3}\right)$; whence, taking (2.9) into account, we obtain

$$
\left\|\theta_{n}\right\|_{L^{\infty}\left(0, T ; L^{1}\left(\mathcal{T}^{3}\right)\right)} \leq K
$$

Furthermore, we can use the renormalized equation (2.12) in order to deduce

$$
\int_{0}^{T} \int_{\mathscr{T}^{3}}\left|H^{\prime \prime}\left(\theta_{n}\right)\right|\left|\nabla_{x} \theta_{n}\right|^{2} \mathrm{~d} x \mathrm{~d} t \leq K,
$$

for any concave increasing $H \in C^{2}[0, \infty)$. In particular, as we already know that $\theta_{n}$ are bounded from below uniformly in $n$, we can choose $H(\theta)=\theta^{\alpha}$ to obtain

$$
\left\|\nabla_{x} \theta_{n}^{\alpha / 2}\right\|_{L^{2}\left(0, T ; L^{2}\left(\mathscr{T}^{3} ; \mathbb{R}^{3}\right)\right)} \leq K
$$

for any $0<\alpha<1$, which, together with (3.10), implies that

$$
\left\|\theta_{n}^{\alpha / 2}\right\|_{L^{2}\left(0, T ; W^{1,2}\left(\mathscr{T}^{3}\right)\right)} \leq K \quad \text { for any } 0<\alpha<1
$$

Now, by virtue of the embedding relation $W^{1,2}\left(\mathscr{T}^{3}\right) \hookrightarrow L^{6}\left(\mathscr{T}^{3}\right)$, the estimate (3.13) yields

$$
\left\|\theta_{n}^{\alpha}\right\|_{L^{1}\left(0, T ; L^{3}\left(\mathscr{T}^{3}\right)\right)} \leq K \quad \text { for any } 0<\alpha<1 .
$$


10 NSEs with temperature-dependent transport coefficients

Using then the interpolation inequality

$$
\|z\|_{L^{q}\left(\mathscr{T}^{3}\right)}^{q} \leq \mathcal{C}\|z\|_{L^{1}\left(\mathscr{T}^{3}\right)}^{(3-q) / 2}\|z\|_{L^{3}\left(\mathscr{T}^{3}\right)}^{3(q-1) / 2},
$$

together with (3.10) and (3.14), we conclude that

$$
\|\left.\theta_{n}^{\alpha}\right|_{L^{5 / 3}\left(0, T ; L^{5 / 3}\left(\mathscr{T}^{3}\right)\right)} \leq K \quad \text { for any } 0<\alpha<1,
$$

which implies

$$
\left\|\theta_{n}\right\|_{L^{q}\left(0, T ; L^{q}\left(\mathscr{T}^{3}\right)\right)} \leq K \quad \text { for any } q \in\left[1, \frac{5}{3}\right) .
$$

Making use of the renormalized equation (2.12) again, this time with $H(\theta)=\theta^{\alpha / 2}$, we conclude from the above estimates that

$$
\left\|\partial_{t} \theta_{n}^{\alpha / 2}\right\|_{L^{1}\left(0, T ; W^{\left.-1, r^{\prime}\left(\mathscr{T}^{3}\right)\right)}\right.} \leq K \quad \text { for any } r>3, r^{\prime}:=\frac{r-1}{r} .
$$

\section{The limit passage for $n \rightarrow \infty$ (M fixed)}

Based on the estimates derived in Section 3 we can select a suitable subsequence of $\left(u_{n}, p_{n}\right.$, $\left.\theta_{n}\right)$ and find $(u, p, \theta):=\left(u_{M}, p_{M}, \theta_{M}\right)$ such that

$$
\begin{gathered}
u_{n} \longrightarrow u \quad \text { weakly in } L^{2}\left(0, T ; W^{1,2}\left(\mathscr{T}^{3} ; \mathbb{R}^{3}\right)\right), \\
u_{n} \longrightarrow u \quad * \text {-weakly in } L^{\infty}\left(0, T ; L^{2}\left(\mathscr{T}^{3} ; \mathbb{R}^{3}\right)\right), \\
\partial_{t} u_{n} \longrightarrow \partial_{t} u \quad \text { weakly in } L^{2}\left(0, T ; W^{-1,2}\left(\mathscr{T}^{3} ; \mathbb{R}^{3}\right)\right), \\
p_{n} \longrightarrow p \quad \text { weakly in } L^{2}\left(0, T ; L^{2}\left(\mathscr{T}^{3}\right)\right), \\
\theta_{n}^{\alpha / 2} \longrightarrow \theta^{\alpha / 2} \quad \text { weakly in } L^{2}\left(0, T ; W^{1,2}\left(\mathscr{T}^{3}\right)\right), \\
\partial_{t} \theta_{n}^{\alpha / 2} \longrightarrow \partial_{t} \theta^{\alpha / 2} \quad * \text {-weakly in } M\left(0, T ; W^{-1, r^{\prime}}\left(\mathscr{T}^{3}\right)\right):=\left(C_{0}\left(0, T ; W^{1, r}\left(\mathscr{T}^{3}\right)\right)\right)^{*} .
\end{gathered}
$$

In addition, we get from $(2.11)$

$$
\theta_{n}^{\alpha / 2}(t, x) \geq\left[\text { ess } \inf _{y \in \mathscr{T}^{3}} \theta_{n, 0}(y)\right]^{\alpha / 2} \quad \text { for a.a. } t \in(0, T), x \in \mathscr{T}^{3} .
$$

As a consequence of the standard Lions-Aubin compactness lemma, (4.1) and (4.2) imply

$$
\mathbf{u}_{n} \longrightarrow \mathbf{u} \quad \text { strongly in } L^{2}\left(0, T ; L^{2}\left(\mathscr{T}^{3} ; \mathbb{R}^{3}\right)\right)
$$


in particular we can assume

$$
\mathbf{u}_{n}(t) \longrightarrow \mathbf{u}(t) \quad \text { strongly in } L^{2}\left(\mathscr{T}^{3} ; \mathbb{R}^{3}\right) \text { for a.a. } t \in[0, T]
$$

Similarly, using an appropriate generalization of the Lions-Aubin result (see Simon [14], e.g.) one concludes from (4.4) and (4.5) that

$$
\theta_{n}^{\alpha / 2} \longrightarrow \theta^{\alpha / 2} \quad \text { strongly in } L^{2}\left(0, T ; L^{2}\left(\mathscr{T}^{3}\right)\right)
$$

This, together with (3.16) and (3.17), yields

$$
\theta_{n} \longrightarrow \theta \quad \text { strongly in } L^{q}\left(0, T ; L^{q}\left(\mathscr{T}^{3}\right)\right) \text { for any } q \in\left[1, \frac{5}{3}\right)
$$

Now, since $\operatorname{div} \mathbf{u}_{n}=0$ we get from (4.1) that

$$
\operatorname{div} \mathbf{u}=0
$$

Note also that $\mathbf{u} \in L^{2}\left(0, T ; W^{1,2}\left(\mathscr{T}^{3} ; \mathbb{R}^{3}\right)\right), \partial_{t} \mathbf{u} \in L^{2}\left(0, T ; W^{-1,2}\left(\mathscr{T}^{3} ; \mathbb{R}^{3}\right)\right)$; whence $\mathbf{u} \in C$ $\left([0, T] ; L^{2}\left(\mathscr{T}^{3} ; \mathbb{R}^{3}\right)\right)$, in particular, the initial condition makes sense.

In view of (4.1)-(4.10), it is a routine matter to take the limit for $n \rightarrow \infty$ in (2.17) to obtain (since $\mathbf{u}, p$, and $\theta$ are depending on $M$ we write, for a later use, $\mathbf{u}_{M}$ instead of $\mathbf{u}$, and so forth in the limit equation)

$$
\begin{gathered}
\partial_{t} \mathbf{u}_{M}+\operatorname{div}\left(\mathbf{u}_{M} \otimes\left[\mathbf{u}_{M}\right]_{|\mathbf{k}| \leq M}\right)+\nabla_{x} p_{M} \\
=\operatorname{div}\left(\mu\left(\theta_{M}\right)\left(\nabla_{x} \mathbf{u}_{M}+\nabla_{x} \mathbf{u}_{M}^{t}\right)\right) \quad \text { in } L^{2}\left(0, T ; W^{-1,2}\left(\mathscr{T}^{3} ; \mathbb{R}^{3}\right)\right), \\
\mathbf{u}_{M}(0)=\mathbf{u}(0)=\mathbf{u}_{0} .
\end{gathered}
$$

Since $\mathbf{u}=\mathbf{u}_{M}$ is an admissible test function in (4.12), we deduce the energy equality of the form

$$
\|\mathbf{u}(t)\|_{L^{2}\left(\mathscr{T}^{3}\right)}^{2}+\int_{0}^{t} \int_{\mathscr{T}^{3}} \mu(\theta)\left|\nabla_{x} \mathbf{u}+\nabla_{x} \mathbf{u}^{t}\right|^{2} \mathrm{~d} x d \tau=\left\|\mathbf{u}_{0}\right\|_{L^{2}\left(\mathscr{T}^{3}\right)}^{2}
$$

On the other hand, it follows from (2.14) that

$$
\left\|\mathbf{u}_{n}(t)\right\|_{L^{2}\left(\mathscr{T}^{3}\right)}^{2}+\int_{0}^{t} \int_{\mathscr{T}^{3}} \mu\left(\theta_{n}\right)\left|\nabla_{x} \mathbf{u}_{n}+\nabla_{x} \mathbf{u}_{n}^{t}\right|^{2} \mathrm{~d} x d \tau=\left\|\mathbf{u}_{0 n}\right\|_{L^{2}\left(\mathscr{T}^{3}\right)}^{2} .
$$

Thus, taking the limit in (4.15) for $n \rightarrow \infty$, using (4.8) and (2.7), and comparing the result with (4.14), we obtain

$$
\lim _{n \rightarrow \infty} \int_{0}^{T} \int_{\mathscr{T}^{3}} \mu\left(\theta_{n}\right)\left|\nabla_{x} \mathbf{u}_{n}+\nabla_{x} \mathbf{u}_{n}^{t}\right|^{2} \mathrm{~d} x d \tau=\int_{0}^{T} \int_{\mathscr{T}^{3}} \mu(\theta)\left|\nabla_{x} \mathbf{u}+\nabla_{x} \mathbf{u}^{t}\right|^{2} \mathrm{~d} x d \tau .
$$


Obviously, relation (4.16) together with hypothesis (1.8) imply that

$$
\nabla_{x} \mathbf{u}_{n} \longrightarrow \nabla_{x} \mathbf{u} \quad \text { strongly in } L^{2}\left(0, T ; L^{2}\left(\mathscr{T}^{3} ; \mathbb{R}^{3} \times \mathbb{R}^{3}\right)\right) \text {. }
$$

By virtue of (4.16), and compactness established for $\mathbf{u}_{n}$ and $\theta_{n}$, specifically (4.7) and (4.10), we can take the limit in (2.6) to conclude that (we write again $\theta_{M}$ instead of $\theta$, etc.)

$$
\partial_{t} \theta_{M}+\operatorname{div}\left(\theta_{M} \mathbf{u}_{M}\right)-\Delta \mathscr{K}\left(\theta_{M}\right)=\frac{\mu\left(\theta_{M}\right)}{2}\left|\nabla_{x} \mathbf{u}_{M}+\nabla_{x} \mathbf{u}_{M}^{t}\right|^{2} \quad \text { in } \mathscr{D}^{\prime}\left((0, T) \times \mathscr{T}^{3}\right) .
$$

Finally, as for any $\Phi \in \mathscr{D}\left((0, T) \times \mathscr{T}^{3}\right)$ the quantity $u_{M} \Phi$ represents an admissible test function in (4.12), we can add the result of such an operation to (4.18) in order to infer that

$$
\begin{aligned}
& \partial_{t}\left(\frac{\left|\mathbf{u}_{M}\right|^{2}}{2}+\theta_{M}\right)+\operatorname{div}\left(\frac{\left|\mathbf{u}_{M}\right|^{2}}{2}\left[\mathbf{u}_{M}\right]_{|\mathbf{k}| \leq M}\right)+\operatorname{div}\left(\left(p_{M}+\theta_{M}\right) \mathbf{u}_{M}\right) \\
& \quad-\Delta \mathscr{K}\left(\theta_{M}\right)-\operatorname{div}\left(\frac{\mu\left(\theta_{M}\right)}{2}\left(\nabla_{x} \mathbf{u}_{M}+\nabla_{x} \mathbf{u}_{M}^{t}\right) \mathbf{u}_{M}\right)=0 \quad \text { in } \mathscr{D}^{\prime}\left((0, T) \times \mathscr{T}^{3}\right) .
\end{aligned}
$$

\section{The limit passage for $M \rightarrow \infty$}

Given the uniform (with respect to $M$ ) estimates established in Section 3, the last part of the proof of Theorem 1.1 is rather standard. Indeed as the approximate solutions $\left(u_{M}, p_{M}, \theta_{M}\right)$, fulfilling (4.11), (4.12), (4.19), and (4.18), are limits of weakly converging sequences, relations (3.1), (3.2), (3.6), (3.8), (3.10), (3.13), and (3.18) remain valid for $\left(u_{M}, p_{M}, \theta_{M}\right)$.

Using the same arguments as in Section 4 , we find subsequences of $\left(u_{M}, p_{M}, \theta_{M}\right)$ and $(u, p, \theta)$ such that

$u_{M} \longrightarrow u \quad$ weakly in $L^{2}\left(0, T ; W^{1,2}\left(\mathscr{T}^{3} ; \mathbb{R}^{3}\right)\right), *$-weakly in $L^{\infty}\left(0, T ; L^{2}\left(\mathscr{T}^{3} ; \mathbb{R}^{3}\right)\right)$,

$$
\begin{gathered}
\partial_{t} u_{M} \longrightarrow \partial_{t} u \quad \text { weakly in } L^{5 / 2}\left(0, T ; W^{-1,5 / 2}\left(\mathscr{T}^{3} ; \mathbb{R}^{3}\right)\right), \\
p_{M} \longrightarrow p \quad \text { weakly in } L^{5 / 3}\left(0, T ; L^{5 / 3}\left(\mathscr{T}^{3}\right)\right), \\
\theta_{M}^{\alpha / 2} \longrightarrow \theta^{\alpha / 2} \quad \text { weakly in } L^{2}\left(0, T ; W^{1,2}\left(\mathscr{T}^{3}\right)\right), \\
\partial_{t} \theta_{M}^{\alpha / 2} \longrightarrow \partial_{t} \theta^{\alpha / 2} \quad \text { weakly in } M\left(0, T ; W^{-1, r^{\prime}}\left(\mathscr{T}^{3}\right)\right) \text { with } r>3 .
\end{gathered}
$$

Moreover, we have

$$
\begin{aligned}
& \mathbf{u}_{M} \longrightarrow \mathbf{u} \quad \text { strongly in } L^{q}\left(0, T ; L^{q}\left(\mathscr{T}^{3} ; \mathbb{R}^{3}\right)\right) \forall q \in\left[1, \frac{5}{3}\right), \\
& \mathbf{u}_{M}(t) \longrightarrow \mathbf{u}(t) \quad \text { strongly in } L^{2}\left(\mathscr{T}^{3} ; \mathbb{R}^{3}\right) \text { for a.a. } t \in[0, T] .
\end{aligned}
$$


In addition, similarly to Section 4 , we can deduce

$$
\theta_{M}^{\alpha / 2} \longrightarrow \theta^{\alpha / 2} \quad \text { strongly in } L^{2}\left(0, T ; L^{2}\left(\mathscr{T}^{3}\right)\right)
$$

leading to

$$
\theta_{M} \longrightarrow \theta \quad \text { strongly in } L^{q}\left(0, T ; L^{q}\left(\mathscr{T}^{3}\right)\right) \text { for any } q \in\left[1, \frac{5}{3}\right)
$$

whence, in particular,

$$
\theta_{M}(t) \longrightarrow \theta(t) \quad \text { strongly in } L^{1}\left(\mathscr{T}^{3}\right) \text { for a.a. } t \in[0, T] .
$$

Observing that

$$
\begin{gathered}
\left(\frac{\left|\mathbf{u}_{M}\right|^{2}}{2}+p_{M}\right) \mathbf{u}_{M} \text { are bounded in } L^{10 / 9}\left(0, T ; L^{10 / 9}\left(\mathscr{T}^{3} ; \mathbb{R}^{3}\right)\right), \\
\theta_{M} \mathbf{u}_{M} \text { are bounded in } L^{q}\left(0, T ; L^{q}\left(\mathscr{T}^{3} ; \mathbb{R}^{3}\right)\right) \quad \forall q \in\left[1, \frac{10}{3}\right), \\
\mu\left(\theta_{M}\right)\left(\nabla_{x} \mathbf{u}_{M}+\nabla_{x} \mathbf{u}_{M}^{t}\right) \mathbf{u}_{M} \text { are bounded in } L^{5 / 4}\left(0, T ; L^{5 / 4}\left(\mathscr{T}^{3} ; \mathbb{R}^{3}\right)\right),
\end{gathered}
$$

we can let $M \rightarrow \infty$ in (4.12), and (4.19) in order to show (with help of (5.6), (5.8), and (5.9)) that (1.15), (1.17) hold in $\mathscr{D}^{\prime}\left((0, T) \times \mathscr{T}^{3}\right)$. Furthermore, as convex functionals are weakly lower semicontinuous, it is easy to see that (4.18) gives rise to $(1.18)$ in $\mathscr{D}^{\prime}((0, T) \times$ $\left.\mathscr{T}^{3}\right)$.

As the remaining statements claimed in Theorem 1.1 are standard (the reader can be referred, e.g., to [10]), the proof is now complete.

\section{Acknowledgments}

The work of the first author was supported by Grant no. 201/05/0164 of CSF (GAČR) as a part of the general research programme of the Academy of Sciences of the Czech Republic, Institutional Research Plan AV0Z10190503. The contribution of the second author to this work is a part of the research project MSM 0021620839 financed by MSMT. The support of CSF, the project GACR 201/05/0164, is also acknowledged.

\section{References}

[1] L. Caffarelli, R. Kohn, and L. Nirenberg, Partial regularity of suitable weak solutions of the NavierStokes equations, Communications on Pure and Applied Mathematics 35 (1982), no. 6, 771831.

[2] L. Consiglieri, Weak solutions for a class of non-Newtonian fluids with energy transfer, Journal of Mathematical Fluid Mechanics 2 (2000), no. 3, 267-293.

[3] L. Consiglieri, J. F. Rodrigues, and T. Shilkin, On the Navier-Stokes equations with the energydependent nonlocal viscosities, Zapiski Nauchnykh Seminarov Sankt-Peterburgskoe Otdelenie. Matematicheskií Institut im. V. A. Steklova. (POMI) 306 (2003), 71-91.

[4] J. Duchon and R. Robert, Inertial energy dissipation for weak solutions of incompressible Euler and Navier-Stokes equations, Nonlinearity 13 (2000), no. 1, 249-255. 


\section{NSEs with temperature-dependent transport coefficients}

[5] D. G. Ebin, Viscous fluids in a domain with frictionless boundary, Global Analysis-Analysis on Manifolds (H. Kurke, J. Mecke, H. Triebel, and R. Thiele, eds.), Teubner-Texte zur Mathematik, vol. 57, Teubner, Leipzig, 1983, pp. 93-110.

[6] G. L. Eyink, Local 4/5-law and energy dissipation anomaly in turbulence, Nonlinearity 16 (2003), no. 1, 137-145.

[7] E. Feireisl, Dynamics of Viscous Compressible Fluids, Oxford University Press, Oxford, 2003.

[8] O. A. Ladyzhenskaya, V. A. Solonnikov, and N. N. Uraltseva, Linear and Quasilinear Equations of Parabolic Type, Trans. Math. Monograph, vol. 23, American Mathematical Society, Rhode Island, 1968.

[9] J. Leray, Sur le mouvement d'un liquide visqueux emplissant l'espace, Acta Mathematica 63 (1934), 193-248.

[10] J. Málek and K. R. Rajagopal, Mathematical issues concerning the Navier-Stokes equations and some of its generalizations, Handbook of Differential Equations: Evolutionary Equations. Vol. II (C. Dafermos and E. Feireisl, eds.), North-Holland, Amsterdam, 2005, pp. 371-459.

[11] T. Nagasawa, A new energy inequality and partial regularity for weak solutions of Navier-Stokes equations, Journal of Mathematical Fluid Mechanics 3 (2001), no. 1, 40-56.

[12] J. Nečas and T. Roubíček, Buoyancy-driven viscous flow with $L^{1}$-data, Nonlinear Analysis 46 (2001), no. 5, 737-755.

[13] T. Shilkin, Classical solvability of the coupled system modelling a heat-convergent Poiseuille-type flow, Journal of Mathematical Fluid Mechanics 7 (2005), no. 1, 72-84.

[14] J. Simon, Compact sets in the space $L^{p}(0, T ; B)$, Annali di Matematica Pura ed Applicata. Serie Quarta 146 (1987), 65-96.

Eduard Feireisl: Mathematical Institute, Academy of Sciences of the Czech Republic, Žitná 25, 11567 Praha 1, Czech Republic

E-mail address: feireisl@math.cas.cz

Josef Málek: Mathematical Institute, Faculty of Mathematics and Physics, Charles University in Prague, Sokolovská 83, 18675 Praha 8, Czech Republic

E-mail address: malek@karlin.mff.cuni.cz 


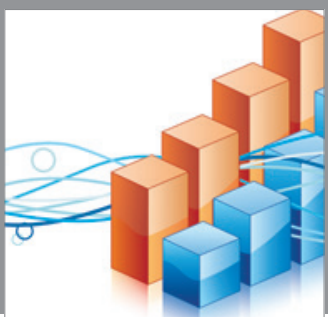

Advances in

Operations Research

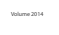

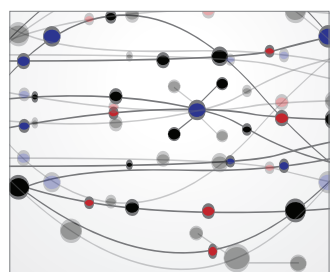

\section{The Scientific} World Journal
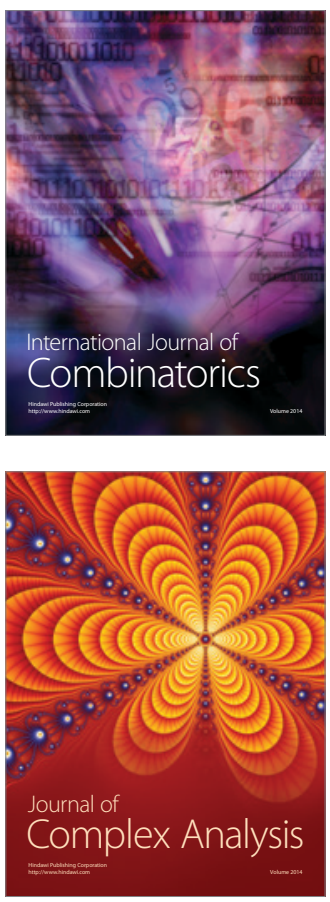

International Journal of

Mathematics and

Mathematical

Sciences
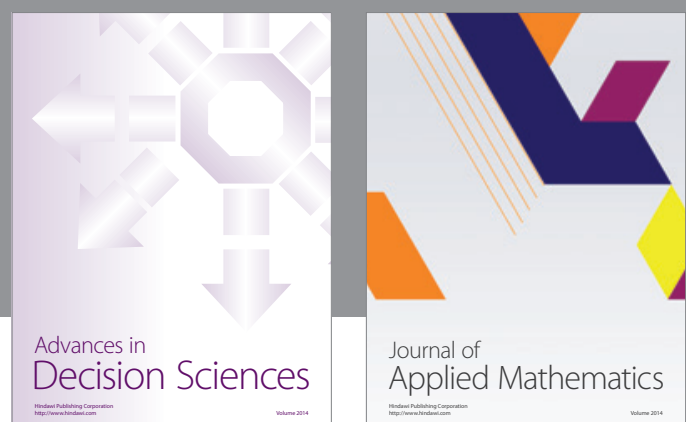

Journal of

Applied Mathematics
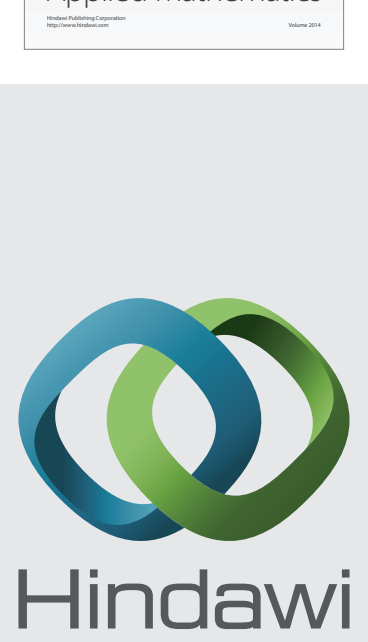

Submit your manuscripts at http://www.hindawi.com
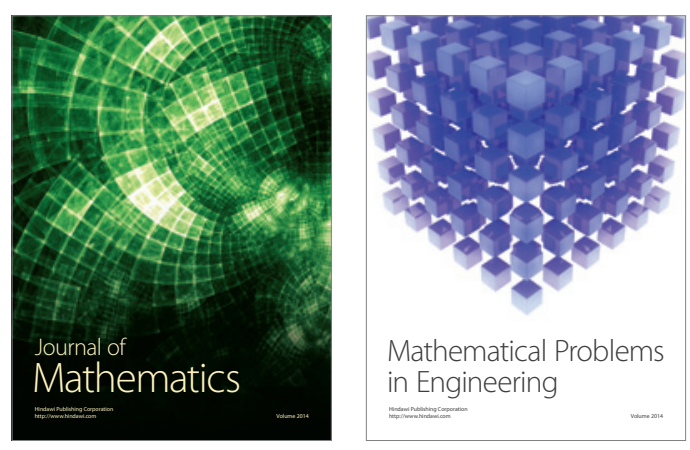

Mathematical Problems in Engineering
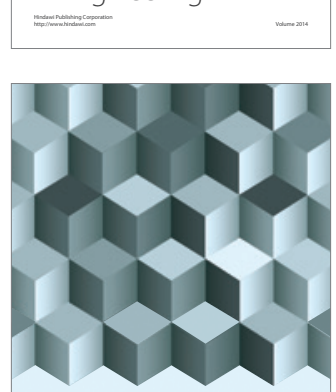

Journal of

Function Spaces
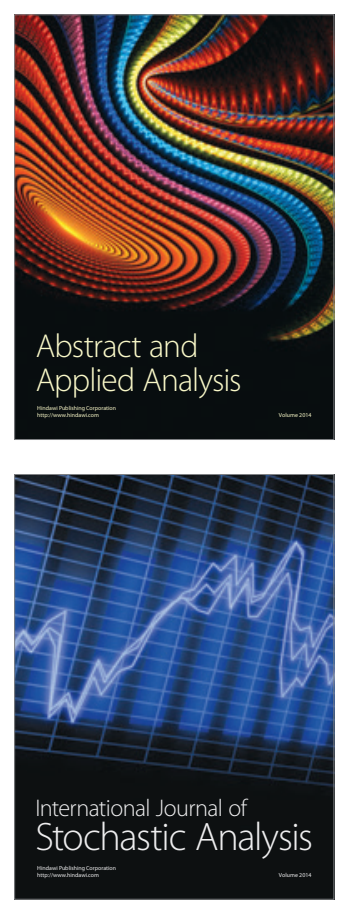

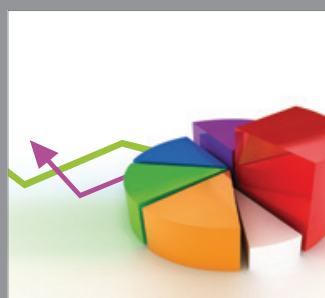

ournal of

Probability and Statistics

Promensencen
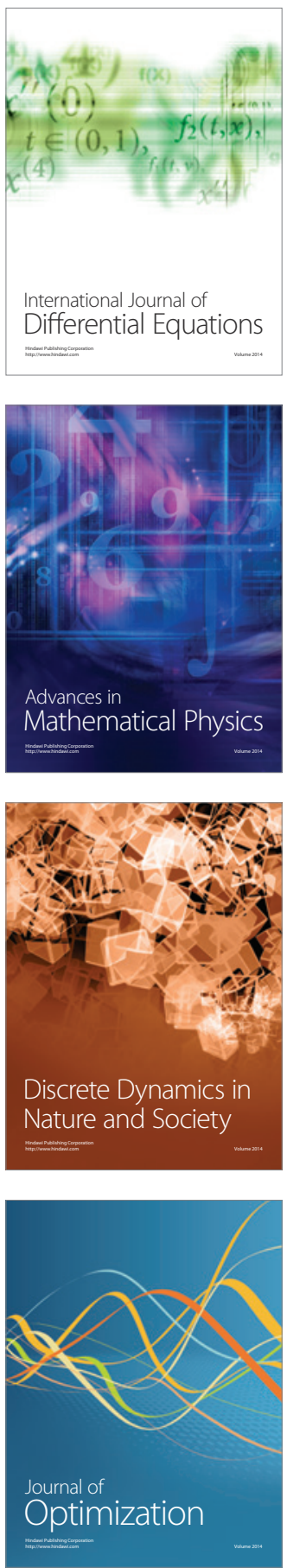